\section{Peter Blumenthal}

Université de Cologne

Allemagne

(iD https://orcid.org/0000-0002-4196-3677

\section{Les mots et les savoirs : complexité}

\title{
Words and knowledge: complexity
}

\begin{abstract}
The contribution addresses a topic discussed since the 17 th century by philosophers, logicians and lexicographers: to what extent does the semantic complexity of certain words convey knowledge of the extralinguistic world? What influence does this knowledge have on the coherence of a text? Conversely, another type of complexity must be taken into account as well, the one starting from things: what linguistic devices are adopted to express this complexity in an efficient way? The relationship between the complexity types and knowledge has been investigated by different strands of research in the humanities and is becoming a focus of multidisciplinary research.
\end{abstract}

Keywords

Knowledge, complexity, coherence, definition

\section{Perspectives pluridisciplinaires de la linguistique}

Dans les recherches d'une discipline, l'histoire, avec laquelle les sciences du langage ont longtemps fait cause commune, apparaissent, depuis le début de ce siècle, de nouveaux centres d'intérêt qui ne devraient pas laisser les linguistes indifférents. Nous pensons plus particulièrement à "l'histoire des savoirs », actuellement en train d'accroître son influence sur les programmes d'enseignement et de recherche universitaires ${ }^{1}$. Certains aspects de cette problématique sont bien

\footnotetext{
${ }^{1}$ Cf. S. Van Damme : «L'histoire des savoirs a 20 ans » dans Le Monde 8/7/2020.
} 
connus des linguistes ${ }^{2}$, parfois même depuis des décennies, comme la genèse des langues de spécialité ou l'histoire des concepts scientifiques. Or, un projet de la dimension des Lieux de savoir (dirigé par Christian Jacob), qui « constitue une réflexion sur la manière dont les savoirs s'élaborent, prennent forme et se transmettent $»^{3}$, demande au linguiste d'autres compétences encore. La tâche fondamentale serait sans aucun doute l'engagement dans une collaboration interdisciplinaire intensifiée, à laquelle la linguistique pourrait contribuer par une conception cognitive de l'histoire de la langue. Nous en parlons au conditionnel, dans l'impossibilité actuelle où nous sommes de dessiner avec netteté les contours d'une telle coopération entre une nouvelle branche de l'histoire (générale) et une diachronie linguistique modernisée. En attendant, nous nous pencherons sur quelques aspects d'un problème central, la complexité ${ }^{4}$ dans la sphère du savoir, dont on peut prévoir, sans être devin, qu'il jouera un rôle important dans une future collaboration interdisciplinaire. Thème à mille facettes, soulevant des questions redoutables, et qu'il faut fortement spécifier pour pouvoir en discuter dans le cadre de la présente contribution. Dans notre approche du problème, dont la seule ambition sera la distinction de quelques cas de figures, nous avons choisi de mettre l'accent sur deux thèmes particuliers qui concernent la langue transportant le savoir :

1. Comment décrire et classifier la complexité sémantique contenue dans les unités lexicales, et surtout les noms, principaux porteurs du savoir véhiculé par la phrase ?

2. Quelles sont les stratégies linguistiques, et avant tout les unités lexicales dont dispose un auteur pour exprimer de manière compréhensible des phénomènes extralinguistiques complexes ? Problème traité par la rhétorique classique dans les chapitres sur la perspicuitas.

Dans les deux cas, l'unité linguistique qui constitue le cadre au déploiement de la complexité est le texte. Après ces quelques observations sur une linguistique de l'avenir, un rapide retour en arrière s'impose - sur un penseur mort en 1716, G. W. Leibniz, à qui nous devons, sinon la découverte de la problématique, du moins des réflexions lucides et profondes, rédigées dans un français dont la lecture, plus de trois siècles après, reste une jouissance.

${ }^{2}$ Cf. les travaux du Centre de Recherches sur l'analyse et la théorie des savoirs de l'Université de Lille III.

${ }^{3}$ « Lieux de savoir », Wikipédia. https://fr.wikipedia.org/wiki/Lieux_de_savoir (consulté le 05.07.2021).

${ }^{4}$ Pour l'histoire de ce concept, cf. E. Morin, $2005: 46-51$. 


\section{Ce qu'apportent les mots selon Leibniz}

Dans son livre Nouveaux essais sur l'entendement humain, Leibniz esquisse une théorie sémantique dans laquelle la discussion (il s'agit en effet d'un dialogue fictif) de ce que l'on pourrait appeler de nos jours " apport cognitif des mots » joue un rôle de premier plan, et cela autour de notions comme " mémoire », « attention ", « composition » et « connaissance ». Le philosophe y soulève des problèmes qui, parfois sous une autre forme, restent d'actualité. Tout d'abord, par sa vision du « signe », qui peut représenter ${ }^{5}$ une ou plusieurs « idées », rapportées à « un ordre naturel ». Ensuite par l'hypothèse qu'un tel assemblage d'idées conditionne le passage à un niveau plus global de connaissance, celle-ci n'étant « autre chose que la perception de la liaison et convenance ou de l'opposition ou disconvenance qui se trouve entre deux de nos idées » (1990: 281). Ces combinaisons d'idées reposent sur des relations (1990 : 176), facteurs d'une complexité qui a « quelque chose de volontaire », car il faut un esprit « actif » pour la créer (1990 : 204). Les idées simples, en revanche, sont en général données telles quelles à l'esprit " passif ». Reste le problème, fondamental pour toute forme d'épistémologie, du rapport entre connaissance et vérité. Leibniz le ramène au « rapport entre les objets et les idées » (1990 : 312). «Cela ne dépend point des langues », affirme-t-il, mais d'une sorte d'intervention divine (1990 : 313).

Ses réflexions sur les rapports entre idées combinées et mots complexes amènent Leibniz à quelques considérations contrastives. Il relève, p. ex., que la fusion de diverses idées dans ostracisme (chez les Grecs) et proscription (parmi les Romains) rendent ces mots difficilement traduisibles (1990:167) :

Il est toujours vrai que, les mœurs et les usages d'une nation faisant des combinaisons qui lui sont familières, cela fait que chaque langue a des termes particuliers [...].

Il existe ainsi des mots-témoins des spécificités d'une civilisation (comme triomphe chez les Romains (1990 : 235)) ${ }^{6}$. Même l'absence de certains mots nous renseigne sur des particularités culturelles; exemple : à côté de parricide, il n'y a pas de mot en français pour l'idée de tuer un vieillard (1990 : 167). Du lien systémique et souvent lexicalisé entre idées il faut distinguer, selon Leibniz le lien associatif, qui se développe à partir de l'expérience individuelle et accidentelle du locuteur?

5 Terme important chez Leibniz. En faisant du signe (appartenant à la langue) le représentant de l'idée (entité mentale), Leibniz ne s'expose pas aux débats que suscite, spécialement dans la psycholinguistique contemporaine, le rapport entre concept et signification ; cf. le chapitre « Concepts and word meanings : do we need to draw a line? », dans G. Vigliocco, D. P. Vinson, 2007 : 196.

${ }^{6}$ Veine habilement exploitée par F. Predazzi, V. Vannuccini, ci-dessous 8.

7 Exemple (1990 : 207) : « On ne pense pas à un homme qu'on hait sans penser au mal qu'il nous a fait $[\ldots] »$. 


\section{Aspects de la complexité}

Cette analyse des idées et de leurs combinaisons, dont le principe nous servira dans un premier temps ${ }^{8}$ de fil conducteur, va de pair, chez Leibniz, avec une réflexion sur l'instrument privilégié de ce genre d'étude qu'est la définition, appliquée p. ex. aux noms d'affect (Livre II, chap. XX). Vu la subtilité de ses observations, notre angle d'attaque, centré sur les définitions et visant les rapports entre lexique et savoirs, risquera de paraître peu prometteur ; nous partirons de l'observation, quelque peu banale, que les définitions des mots, et surtout celles des noms, données par les dictionnaires se distinguent fortement par leur longueur. À titre d'exemple, voici deux définitions de noms choisis au hasard dans le Petit Robert (désormais PR). Un chapeau est une «Coiffure de forme le plus souvent rigide (opposé à bonnet, coiffe) »(PR, chapeau I.). La définition du nom désignant le genre prochain (coiffure) n'apporte guère plus de détails : « Ce qui sert à couvrir la tête ou à l'orner » (PR, coiffure 1.). En revanche, la définition - aux allures encyclopédiques - d'oiseau est presque 5 fois plus longue que celle de coiffure :

(1) Animal appartenant à la classe des vertébrés tétrapodes à sang chaud, au corps recouvert de plumes, dont les membres antérieurs sont des ailes, les membres postérieurs des pattes, dont la tête est munie d'un bec corné dépourvu de dents, et qui est en général adapté au vol.

(PR, oiseau I.1.)

Certes, oiseau et coiffure appartiennent à des domaines trop différents pour se prêter à des comparaisons sémantiques, mais les profondes différences entre les définitions dictionnairiques donnent à réfléchir. Même des mots sémantiquement plus proches se distinguent souvent par le degré de complexité de leurs définitions, par exemple les deux mots d'affect haine et jalousie. Ainsi, la haine peut n'impliquer que deux êtres ; cf. PR, haine :

(2) Sentiment violent qui pousse à vouloir du mal à qqn et à se réjouir du mal qui lui arrive,

alors que le sens le plus courant de jalousie met en jeu trois personnes ; cf. PR, jalousie I.3. :

(3) Sentiment douloureux que font naître, chez la personne qui l'éprouve, les exigences d'un amour inquiet, le désir de possession exclusive de la personne aimée, la crainte, le soupçon ou la certitude de son infidélité.

${ }^{8}$ Pour des raisons de lisibilité, nous présenterons d'autres types d'approches plus bas dans les paragraphes concernés. 
D'autres noms d'affect ne présupposent que la présence d'une seule personne (cf. tristesse, joie, ennui). Mais pour déterminer la complexité inhérente au sémantisme d'un mot, il faut tenir compte non seulement des personnages mis en scène, mais aussi des circonstances (causes, conséquences, situations antérieures, etc.) qui confèrent leurs rôles aux personnages et auxquelles renvoie le mot, explicitement ou non. Si, par exemple, la haine désigne un affect « qui pousse à vouloir du mal » (v. ci-dessus), il convient de retenir, à côté des actants, le rôle causal de l'affect. Le sémantisme de jalousie comporte également un facteur " causal » (au sens large), exprimé par que font naître, mais en l'occurrence, le sentiment (jalousie) n'est pas cause, mais résultat.

Dans le cas des noms d'affect, nous proposons de retenir, en tant qu'éléments de la complexité sémantique, le nombre des « actants » et des « circonstants 》 (selon la terminologie et le modèle syntaxique de L. Tesnière (1969)). Toutes choses étant égales d'ailleurs, nous considérons que la complexité de la définition, donc du sémantisme du mot en question, croît proportionnellement avec le nombre des actants et circonstants.

À la fin de ce paragraphe, force est de reconnaître (à titre de «prolepse » ou d'« anticipation », aux termes de la rhétorique) que certaines questions restent ouvertes, concernant :

- la comparabilité des définitions, qui varient souvent d'un dictionnaire à l'autre ;

- l'aptitude des définitions à représenter le sémantisme du $\operatorname{mot}^{9}$;

- des contradictions inhérentes au modèle de Tesnière, dont les critères pour la distinction d'actants et de circonstants ne convainquent pas toujours ${ }^{10}$.

Face à ces problèmes, force est d'admettre qu'en l'occurrence, la perspective d'explorer des approches possibles l'emporte sur des scrupules de détail.

Mentionnons dès maintenant pour mémoire une question discutée plus bas (11.1) à propos du modèle syntaxique. En plus de ce que Tesnière appelle, dans l'analyse de la phrase, les « actants » et les « circonstants », on peut identifier, dans les définitions, une troisième source de complexité, à savoir les éléments de classification ou de distinction. Lorsque le mot à définir représente une substance (« oiseau » dans l'exemple ci-dessus), la classification se fait en général par le biais d'adjectifs épithètes (ex. : tétrapodes), de relatives restrictives ou de compléments déterminatifs (à sang chaud); en cas d'accumulation de telles constructions, le linguiste s'interrogera sur la limite entre les distinctions structurellement indispensables et les indications relevant d'une vision plutôt descriptive ou prototypique (cf. « en général adapté au vol ») du mot.

${ }^{9}$ Comme l'explique Rey-Debove (1971: 195), la définition « est une explicitation naturelle dont la valeur, c'est-à-dire l'utilité réelle, est cautionnée par le lecteur moyen (non linguiste). Or le lecteur moyen n'a aucun besoin d'une description sémantique totale qui soit juste et précise. »

${ }^{10} \mathrm{Cf}$. la discussion dans G. Gross, 2012 : 11, 51-54. 


\section{Complexité, connexité et cohérence textuelle}

Comme nous venons de le voir dans le sillage de Leibniz, la complexité du mot a une dimension cognitive, car la connaissance du mot équivaut, pour le locuteur moyen, à la capacité de saisir un segment de la réalité. Mais la complexité implique aussi une pertinence textuelle, puisque les éléments du sémantisme d'un mot ont de fortes chances de rencontrer des corrélats dans le contexte de ce mot. Il paraît, en effet, peu probable qu'un récit contenant le mot jalousie - pour reprendre l'exemple ci-dessus - ne fasse pas mention des actants présupposés par ce nom, sans forcément les identifier. Le linguiste peut analyser ce phénomène sous divers aspects : selon le point de vue choisi, il s'agit d'anaphores, d'isotopies, de schèmes sémantiques, de frames, etc. Nous préférons parler de " connexité », en étendant toutefois ce concept psycholinguistique (en anglais connectivity) à la dimension textuelle. Nous retiendrons ce terme en raison de son emploi dans les études de psychologie associative (plus bas, 8.). Selon Traxler (2012 : 87), " connectivity reflects how many words are associated with a specific target word and how many connections are shared between that set of words. » Nous considérons, avec Traxler (2012 : 87), que le type de connexité (low vs high connectivity) fait partie des caractéristiques du mot. Dans la tradition lexicographique française, les dictionnaires analogiques peuvent passer pour les plus vieilles sources d'information sur la connexité. Grâce aux méthodes stylistiques appliquées aux corpus, nous disposons aujourd'hui d'outils plus fiables pour saisir sous forme de "profil combinatoire " la connexité des mots, que nous considérons comme corrélée à la complexité.

\section{Aux sources de la complexité}

D'après notre hypothèse, la connexité des mots favorise en principe la cohérence du texte - toutefois, sans en constituer une condition nécessaire (cf. F. Neveu, 2011, connexité). Pour mieux comprendre l'enchaînement qui va de la complexité sémantique au texte, en passant par la connexité, il importe d'étudier les détails de la complexité, condition première, et de s'interroger sur ses composantes, en particulier sur l'aspect relationnel : quelles relations existe-t-il entre les arguments et les circonstants contenus dans les définitions données par les bons dictionnaires ? Le PR électronique met à disposition des programmes permettant une réponse empiriquement fondée. Pour bien préparer le travail, il est utile de lire d'abord quelques centaines de définitions choisies au hasard dans ce même dictionnaire. C'est ainsi que l'on peut se faire une idée approximative des types de 
relations et des mots qui les articulent dans la tradition du dictionnaire consulté. Ainsi, la définition de antisismique ("Conçu pour résister aux séismes ») comporte, du moins implicitement, deux arguments ${ }^{11}$ et une relation finale exprimée par pour, comme d'autres mots avec le même préfixe. Ensuite, à l'aide du menu du PR (ou celui du TLFi), on fait rechercher toutes les définitions contenant le mot voulu. Les relations à l'intérieur des définitions s'avèrent, sans grande surprise, surtout temporelles et/ou causales (au sens large). Voici quelques indications de fréquences de mots figurant dans les définitions :

motivé : 12 ; afin : 161 ; raison : 177 ; d̂̀ à : 191 ; suite : 327 ; cause : 375 ; avant : 534 ; après : 707.

Les emplois de la préposition pour, très fréquents $(4754 \mathrm{x})$ et souvent finaux, nécessiteraient une explication au cas par cas pour déterminer leurs valeurs relationnelles exactes.

Certaines locutions avec suite se font remarquer par la fusion qu'elles opèrent entre les catégories de l'espace, du temps et de la cause, ensemble que le lexicographe a du mal à disséquer; cf. la citation suivante (PR, suite B.2.) :

À LA SUITE DE (dans l'espace) : en suivant derrière, en se faisant suivre par-derrière. [...] - Derrière, en considérant un ordre donné. [...] - (Dans le temps) Après, en suivant. [...] - Spécialt. (l'événement suivant sa cause) À cause de, en raison de. $I l$ « s'était fait prêtre, à trente-deux ans, à la suite d'un chagrin d'amour ».

Un autre type de fusion dans la définition ne porte pas sur les relations, mais sur les entités (en fonction d'actants ou de circonstants, v. plus bas) ; que l'on observe la définition suivante de dépit dans le PR :

(4) Chagrin mêlé de colère, dû à une déception personnelle, un froissement d'amour-propre.

On peut concevoir ce " mélange », facteur de complexité, comme un cas de simultanéité. Grâce au menu du PR, on retrouve facilement d'autres noms d'affect définis comme un mélange, à savoir amertume, confusion, crainte et trouble.

Ce genre d'analyse, appliquée aux mots complexes d'un texte, renseigne sur les relations et les autres mots qui se présentent avec une certaine probabilité dans le contexte, alors que la recherche statistique dans l'ensemble des définitions d'un dictionnaire fait apparaître les principales composantes de la complexité sémantique.

${ }^{11}$ On pourrait penser à construction et séisme. 


\section{6. Étude de cas}

Pour illustrer le mouvement de structuration allant d'un mot au texte entier, nous avons choisi un article de journal où le mot complexe (dépit) figure dans le titre. L'article thématise et développe ce concept dans les phrases successives, en reprenant ses différents aspects (renvoi aux noms d'affect et à une circonstance causale). Le choix initial de dépit conduit donc à la sélection de quelques contenus impliqués par ce mot. Voici l'exemple à discuter :

(5) Par dépit, la lycéenne balafre sa rivale

Elle était amoureuse. Et sa meilleure copine de classe lui a "piqué 》son petit copain. La jeune fille de 18 ans, scolarisée dans un lycée rennais, n'a pas supporté l'affront. Elle a boudé son amie pendant une dizaine de jours. Puis sa colère est montée crescendo. Mardi dernier, la jeune femme arrive au lycée, armée [...].

(Ouest-France, 2008)

Ce fait divers présente, directement ou de façon paraphrastique, la totalité des traits sémantiques mentionnés par le PR dans la définition de dépit (v. ci-dessus (4)) ; ainsi, « froissement d'amour-propre » correspond à affront, " chagrin » à n'a pas supporté, alors que « colère " se trouve tel quel dans le texte. L'idée de dépit suscite ici l'image d'une $«$ tranche de vie $»^{12}$, qui se voit arrondie par un état que l'on pourrait qualifier de pré-initial (" elle était amoureuse ») et par une action finale résultant de la colère : l'attaque de la rivale. Voilà une belle illustration d'un possible rapport entre complexité du mot et cohérence du texte.

\section{Complexité et locutions}

Il convient de distinguer de la complexité sémantique du mot (exemple : dépit) celle des locutions idiomatiques, qui constituent des unités polylexicales plus ou moins figées. Elles sont complexes dans la mesure où elles impliquent ou évoquent une pluralité de relations, ce qui leur permet souvent de contribuer à la cohésion du texte. Prenons pour exemple ${ }^{13}$ l'expression couper l'herbe sous

12 «Une tranche de vie, ou slice of life est, dans le langage courant, une petite séquence de la vie d'un être caractérisée par un événement particulier, anecdotique ou capital », Wikipédia. https:// fr.wikipedia.org/wiki/Tranche_de_vie (consulté le 20.06.2021).

${ }^{13}$ Cf. P. Blumenthal, S. Mejri, $2019: 299$. 
le pied à qqn, que le Petit Robert (sous herbe) paraphrase par 'frustrer qqn d'un avantage en le devançant, en le supplantant'. Cette locution évoque, en guise de schème, une suite d'étapes comprenant une case départ (antagonisme entre au moins deux personnes) et une action habile (phase centrale) de l'une des personnes, qui finit par s'imposer au détriment de l'autre. La complexité des états des choses peut correspondre à une succession d'événements relatés (et d'autant de relations), dont elle représente un condensé - rhétoriquement utile au début d'un récit, à l'un de ses tournants ou à la fin en tant que rétrospective, donc aux positions stratégiques du texte. L'effet de cet emploi d'une locution peut se déployer en trois phases : a) réception de la locution par l'auditeur ; b) émergence chez l'auditeur d'une attente schématique, conforme au sens de la locution; c) concrétisation, dans le texte, de l'information spécifique et actualisée. Le locuteur fait passer l'attention de l'auditeur et la " thématisation $»^{14}$ d'un niveau abstrait, celui de la locution sémantiquement complexe, au niveau concret et compositionnel du message proprement dit.

L'avantage communicatif du recours à une locution réside sans doute dans l'orientation holiste donnée au passage entier qui apparaît comme la réalisation particulière d'une situation globale standard, connue de l'auditeur. La locution permet de subsumer l'information spécifique et rhématique sous une schématisation en général connue.

\section{Complexité et mots composés}

Le caractère complexe et le sens de la majorité des mots composés, interprétables conformément au principe de la compositionnalité ${ }^{15}$, se révèlent immédiatement et en dehors d'un système de règles spécifiques. Ainsi, des unités lexicales comme porte-clés ('anneau pour porter des clés'), femme de ménage ou essuieglace sont interprétées comme des condensations nominales de phrases ou de syntagmes, dont elles conservent pour l'essentiel le sens. Or, les exceptions ne manquent pas ; sage-femme, par exemple, n'est pas l'abrégé de femme qui est sage. Pour que l'unité complexe soit reconnue en tant que telle par la communauté linguistique et, le cas échéant, lexicalisée, il faut l'accord, au moins tacite, de la part du souverain qu'est l'usage ; celui-ci peut statuer qu'une nouvelle combinaison ne s'assimile pas à une création $a d$ hoc, mais à la représentation d'un phénomène réel et assez typique pour mériter une dénomination spéciale. Toutefois, il faut admettre qu'il émerge parfois, dans l'espace public, des formations (volontai-

${ }^{14}$ Cf. A. Greimas, J. Courtés (1979) sous Thématisation.

${ }^{15}$ Le sens d'une expression résulte du sens de ses composants. Cf. F. Neveu (2011), compositionnalité. 
rement ?) curieuses dont on ne voit ni typicité ni même réalité, et dont les promoteurs ne se comportent pas forcément en protagonistes de l'usage. Description qui vaut, selon nous, pour l'expression islamo-gauchisme qui a défrayé la chronique fin 2020/début 2021. D'autres compositions encore s'écartent du principe d'une interprétabilité immédiate par les aléas de leur évolution historique ou rhétorique (p. ex. couvre-feu).

Sur plusieurs des points mentionnés ci-dessus, ces mots ne se comportent pas de la même manière en allemand et en français ${ }^{16}$. Car d'une part, le locuteur allemand se sent plus libre de composer, par goût personnel, les mots comme bon lui semble - pratique courante tolérée par l'usage, pourvu que le phénomène désigné ainsi apparaisse comme «typique » (Eichinger, $2000: 45$ ). L'auteur cité paraphrase typique par saillant et évident (2000:120). Nous aurions tendance à préciser typique par des antonymes comme accidentel, isolé ou unique. Pour reprendre un exemple souvent discuté : une Putzfrau (littéralement 'femme qui nettoie' ; donc 'femme de ménage') n'est appelée ainsi que quand elle pratique cette activité professionnellement et durablement ${ }^{17}$.

D'autre part, on rencontre en allemand des compositions dans lesquels les relations entre les mots ne se réduisent pas aux structures syntaxiques de base (actancielles, circonstancielles), comme cela se produit $\mathrm{p}$. ex. pour porte-clés ou Putzfrau. En effet, le sens total d'un mot composé en allemand peut aller largement au-delà de ce que signifierait normalement la somme sémantique des deux ou trois mots reliés à l'aide de relations syntaxiques. Dans le cas d'un tel ensemble sémantique, « la connaissance du tout et de ses lois ne saurait être déduite de la connaissance séparée des parties qu'on y rencontre » (A. Lalande, 1972 : 373). La plupart du temps, l'on croit pouvoir se contenter, pour expliquer le phénomène sémantique en question, d'un renvoi générique à la Gestaltpsychologie - à laquelle on risque de trop demander en matière de langue. En réalité, le sens de ces mots composés résulte d'une interaction sémantique intense de leurs composantes, productrice de ce que nous appellerons $"$ plus-value sémantique $»^{18}$. Cette interaction fait largement appel à des connaissances encyclopédiques spécifiques et rend le mot apte à exprimer des états de choses d'une grande complexité. Celle-ci, en combinaison avec la typicité de noms composés discutée plus haut, peut rapprocher certains de ces mots du statut des noms propres. La spécificité de l'apport encyclopédique se nourrit en général de la référence implicite à des situations réelles arrivées dans le passé.

Toutes ces qualités indiquées (typicité, statut intermédiaire entre noms communs et noms propres, apport encyclopédique) font que quelques-uns de ces mots

\footnotetext{
${ }^{16}$ Pour le français, G. Gross, 2012 : 219-232.

${ }^{17}$ En d'autres termes, non moins vagues, les noms en question contiennent des traits qui renvoient à leur caractère essentiel et à une valeur aspectuelle. Pour une vision contrastive de la composition en allemand et français, cf. Cassin, 2004 : 229 (J.-P. Dubost est l'auteur de l'excellent article).

${ }^{18}$ Supplément sémantique qui va au-delà de la somme sémantique de mots combinés.
} 
composés à plus-value se prêtent excellemment à des emplois visant à évoquer des phénomènes spécifiques de l'histoire d'un pays ou d'une civilisation. À cet égard, le français ne dispose pas des mêmes facilités que l'allemand. Pour s'en convaincre, on peut se contenter d'un exemple parlant : un certain mouvement réformateur qui s'est constitué à la fin du XIX ${ }^{\mathrm{e}}$ siècle s'appelle Jungtürken en allemand, et non pas Junge Türken, qui réfèrerait simplement à la jeunesse turque tout en étant privé de la plus-value apportée par le composé. En revanche, le français ne saurait signaler cette valeur que par l'orthographe (majuscule et tiret) : les Jeunes-Turcs.

Vu les qualités des mots composés en allemand, il n'est pas étonnant que dans leur livre sur l'âme allemande, deux journalistes italiennes accréditées à Berlin aient choisi un mot composé allemand comme titre de chaque chapitre, en essayant de le traduire tant bien que mal en italien. Dans la traduction française (F. Predazzi, V. Vannuccini), la table des matières se présente pour l'essentiel comme suit :

(6) La vision du monde (Weltanschauung)

Marcher quand l'autre marche (Mitläufer)

Le souilleur de nid (Nestbeschmutzer)

Le repos du soir (Feierabend)

Le penseur latéral (Querdenker)

Celui qui veut toujours avoir raison (Rechthaber)

Se réjouir des malheurs d'autrui (Schadenfreude)

Femmes de quotas (Quotenfrauen)

La solitude à deux (Zweisamkeit)

Le chemin de randonnée (Wanderweg)

La maîtrise du passé (Vergangenheitsbewältigung)

L'amitié virile (Männerfreundschaft)

L'union d'intérêt (Zweckgemeinschaft)

Le non-mot (Unwort)

L'esprit des temps (Zeitgeist)

Ces composés n'appartenant pas tous à la même catégorie, nous procéderons pour l'analyse à la sélection de quelques items particulièrement instructifs. Signalons préalablement une difficulté d'ordre sociolinguistique : le type d'emploi et l'interprétation de certains de ces mots dépendent largement de l'arrière-plan socioculturel et de l'âge des locuteurs et sont, par conséquent, susceptibles de variation et - dans la bouche de la même personne - de fluctuation. Cette caractéristique ressort rapidement de la moindre enquête que l'on peut mener autour de soi. En simplifiant, on peut dire que les variétés du sémantisme s'étalent entre deux bornes : celle $(=\mathrm{A})$ d'une compréhension compositionnelle incluant l'idée de typicité et celle ( $=\mathrm{B})$ d'une prise en compte globale des connaissances ency- 
clopédiques, conduisant à une forte connexité. Entre $\mathrm{A}$ et $\mathrm{B}$, il faut imaginer une échelle de spécification croissante du sémantisme, qui intègre de plus en plus d'informations spatio-temporelles et personnelles. Ainsi, la (très improbable) interprétation A de Männerfreundschaft ne contiendrait que le renvoi à une amitié entre personnes de sexe masculin, sans autre implication, ce qui laisserait le lecteur sur sa faim. À l'opposé, le sémantisme selon B se signale par une grande richesse d'informations supplémentaires (de statut sensiblement différent), dont les suivantes :

- Qualité rhétorique (emphatique, hyperbolique) de Männer ('de vrais hommes') ;

- Valeur associative ou connotative (« amitié entre patriarches faite de peu de discours et d'une estime réciproque », F. Predazzi, V. Vannuccini, 2007 : 123);

- Éléments de la mémoire historique collective des Allemands : les Männerfreundschaften du chancelier Kohl avec Gorbatchev et Mitterrand, qui ont changé le cours des choses en Europe ;

- La conscience métalinguistique que le mot et le phénomène ne sont plus de notre temps.

De nos jours, ce mot, devenu rare, peut certes servir à caractériser un type de relations un peu démodé entre les puissants de ce monde, mais il constitue surtout le point de départ d'une chaîne d'associations encadrée par des connaissances historiques et jalonnée par certaines images restées célèbres. Ce constat va à la rencontre de notre hypothèse que certains composés sont aptes à stocker des connaissances et, partant, porteurs de complexité.

Les deux auteures $(2007$ : 124) semblent considérer qu'il n'existe pas de pendant féminin direct à Männerfreundschaft; or, la riche documentation dans google.fr montre le contraire, du moins pour l'époque actuelle : le mot Frauenfreundschaft ('amitié entre femmes') se porte très bien, entre autres dans la presse féminine, qui thématise volontiers la question de la " sincérité » et des confidences entre amies. On voit donc que la comparaison du sémantisme des mots composés Männerfreundschaft et Frauenfreundschaft, appartenant toutes deux au pôle $\mathrm{B}^{19}$, renseigne pertinemment sur les représentations largement asymétriques du masculin et du féminin dans l'imaginaire d'une communauté linguistique. Heureusement qu'il existe les Ferienfreundschaften (amitiés faites pendant les vacances) pour réconcilier tout le monde..., mais incidemment peut-être aussi pour consoler les Quotenfrauen, les femmes auxquelles l'on reproche de n'avoir obtenu leur poste que grâce à un règlement parfois compliqué et contesté juridiquement sur les quotas. Aux antipodes des Quotenfrauen se trouvent, dans l'estime populaire, les fameuses Trümmerfrauen (souvent traduit par femmes des ruines ; littéralement femmes des décombres), glorifiées par de très nombreux

${ }^{19}$ En revanche, les panneaux affichant FRAUENPARKPLATZ (réservé aux femmes), nombreux surtout dans les grands parkings des supermarchés allemands, contiennent un sémantisme de type A. Pour quand le Männerparkplatz? 
monuments. Comme l'explique l'article de Wikipédia ${ }^{20}$, ce terme désigne « les femmes qui, après la Seconde Guerre mondiale, aidèrent à débarrasser les villes des ruines des bâtiments qui avaient été bombardés ». Ces femmes, dont le rôle, l'existence et le cadre spatio-temporel se trouvent nettement circonscrits par des données d'ordre historique, ont acquis un statut mythique dans la mémoire collective allemande. On peut se demander si la référence du mot, par sa notoriété et ses nombreuses représentations sous forme de monuments, ne s'impose pas comme primordiale, aux dépens de sa signification. Dans ce cas, à l'instar de Männerfreundschaft, il s'approcherait du statut d'un nom propre. Or, en l'absence de critères sûrs et reconnus, cette hypothèse nécessite confirmation. Cela vaut aussi pour Vergangenheitsbewältigung, autre mot-clé de l'après-guerre (et au-delà). La plupart du temps, ce terme ne signifie pas ce qu'il dit littéralement et ce qui correspondrait à un hypothétique sens A ('maîtrise du passé'), mais réfère très spécifiquement à une réflexion sur le passé nazi de l'Allemagne, et en particulier sur l'Holocauste. Predazzi et Vannuccini (2007 : 112) soulignent que cette notion « fut omniprésente dans les programmes scolaires, à la page littéraire des journaux, dans les débats télévisés de la radio nationale ». Cette description donne une idée de la complexité des connaissances liées à ce mot, dont on ne saurait surestimer l'impact sur la civilisation allemande d'après-guerre. Elle confirme par là un riche potentiel de spécification, transformable en plus-value sémantique, qui caractérise de nombreux mots composés en allemand.

Il est apparu que les composés, allemands ou français, se distinguent toujours de la formulation syntaxiquement explicite par une plus-value au moins minimale. En allemand, le volume de cette plus-value peut varier fortement d'un mot à l'autre, partiellement en fonction du type de composition. Pour certains mots composés, la plus-value consiste en un enrichissement important des connaissances ou des tranches de l'imaginaire collectif qu'ils représentent. Ainsi deviennent-ils des réservoirs d'informations à usage multiple : ils semblent prédestinés à stocker et conserver un savoir complexe, servent de charnière entre connaissances linguistiques et encyclopédiques et peuvent contribuer à la cohérence du texte (M. Kauffer, 2016).

En résumé, certains mots composés traités ci-dessus se caractérisent par

— une complexité sémantique à géométrie variable ; les interlocuteurs peuvent s'accommoder, par exemple, d'un sens relativement superficiel et général de Männerfreundschaft, ou bien, au contraire, mettre à profit les trésors de connaissances que véhicule ce mot ;

— un impact fortement différencié sur la cohérence du texte, en fonction des composantes sémantiques du mot actualisées.

${ }^{20}$ Sous Femmes des ruines, https://fr.wikipedia.org/wiki/Femmes_des_ruines (consulté le 12.06.2021). Predazzi et Vannuccini ne traitent pas ce mot. 
La dimension qui encadre la modulation de la complexité de ces composés est celle d'un axe que l'on peut imaginer vertical, allant du général (en haut) à la spécification (en bas).

\section{Complexité et abstraction}

En 6., nous avons mentionné l'une des fonctions des locutions qui peuvent faciliter pour le lecteur la compréhension d'un texte exposant une situation complexe. Dans ce paragraphe, nous allons aborder une autre stratégie textuelle visant, elle aussi, à maîtriser la complexité extralinguistique, et cela par le jeu de différents degrés d'abstraction : l'auteur résume plusieurs données et circonstances ambiantes en un seul mot, forcément abstrait (comme situation, problème, crise ou question), qui se trouve opposé au nom du protagoniste ou, plus généralement, d'une personne appelée à réagir. Pour désigner cette confrontation, il existe un nombre réduit de tournures qui ont connu un essor quantitatif très important dans la presse du XX' $\mathrm{XX}^{\mathrm{e}}$ siècle, comme face à, en face de, faire face à. Ainsi les aspects multiples et enchevêtrés d'un certain état des choses sont-ils ramenés, souvent par une forte simplification, à une opposition binaire, articulée de façon succincte (type : face à cette situation, le président a décidé... ; cf. P. Blumenthal, 2018). Par leur capacité à couvrir une vaste thématique tout en restant succinctes, ces formules se prêtent parfaitement à divers emplois dans les titres des articles de journaux ; voici un exemple du journal Le Monde (21/5/2021) :

(7) Les partis de gauche divergent sur la ligne à adopter face au malaise des forces de l'ordre (titre, p. 13),

variation du gros titre de la première page qui commence par Face au malaise policier. Ce qui se cache derrière malaise, terme général et en l'occurrence un peu vague, est longuement analysé aux pages 12/13 et figure de nouveau dans l'éditorial de la page 32. Le recours à l'abstraction, représentée ici par malaise, est certainement l'une des méthodes les plus traditionnelles pour rendre accessible un texte qui renvoie à une réalité complexe. Devant cette tâche, la presse écrite a développé au $\mathrm{XX}^{\mathrm{e}}$ siècle des stratégies apparemment efficaces, comportant entre autres l'emploi de nouvelles locutions prépositionnelles et la mise au point d'un microsystème de noms utiles (autour de situation) qui ont probablement permis d'améliorer la lisibilité des articles. 


\section{Sémantique et théories de la connaissance}

Dans l'introduction (plus haut, 1.), nous avons prudemment évoqué la perspective de recherches pluridisciplinaires à propos du thème des savoirs. Le moment est venu d'apporter quelques éclaircissements, non moins prudents. Au cours de cette contribution, nous avons débattu d'une qualité des mots sémantiquement complexes : leurs capacités cognitives comme lieux de stockages et de transmissions des connaissances. Cette propriété, fortement mise en relief par Leibniz, soulève tant de problèmes épistémologiques et sémantiques que nous sommes amené à opérer un choix : nous allons nous limiter au cadre thématique de ce qui est couramment appelé « théories de la connaissance » (L. Soler, 2019 : 31). Selon Besnier, cette branche de la philosophie cherche à « évaluer la part qui revient au sujet et à l'objet dans la constitution d'un savoir » (2016:25); connaissance désigne ici la connaissance ordinaire, non pas celle scientifique, objet de l'épistémologie au sens restreint (U. Eco, 1997 : 52—59 ; M. Blay, 2003 : 178 ; B. Cassin, 2004 : 361—365 ; L. Soler, 2019 : 32).

Les hypothèses actuelles dans ce domaine remontent en général au mathématicien et philosophe B. Russell (1912), que nous citerons d'après la traduction française par F. Rivenc (1989). Russell distingue d'abord deux types de connaissances :

1. connaissance des vérités ou connaissance propositionnelle (« savoir que telle chose est le cas $»)$;

2. connaissance des choses sur la base d'une «expérience directe ».

Alors que l'anglais utilise to know dans les deux cas, d'autres langues différencient, comme le fait remarquer Russell (1989: 66) : " Cette distinction est proche de l'usage habituel des mots savoir et connaître pour le français, wissen et kennen en allemand. » Mais à côté de l'expérience directe (« acquaintance »), il existe une autre forme de connaissance de l'objet, celle " par description », c.-à-d. par l'attribution d'une caractéristique à un objet auquel nous faisons référence. Ainsi, l'objet Bismarck peut se décrire, entre autres, par la qualité « le premier Chancelier de l'Empire allemand »(B. Russell, 1989 : 78). De ces quelques concepts utilisés dans la théorie de la connaissance, nous reviendrons à la linguistique proprement dite, pour examiner d'éventuelles affinités entre les problématiques des deux disciplines, et en particulier entre des types de connaissances et des types de significations. Comme on vient de le voir, les emplois des verbes savoir et connaître servent souvent de critère dans les argumentations d'ordre " épistémologique » (au sens large). Or, cet emploi, très variable au cours des époques de la langue, ne vient se stabiliser en français de France qu'au siècle dernier (cf. P. Blumenthal, 1999). Pour s'assurer de la fiabilité de ce critère, il convient donc de définir les propriétés des deux verbes sur lesquelles reposent les différences d'ordre épistémologique. Russell (1989 : 80 ; cf. A. Lalande, 1972 : 
172) signale lui-même un phénomène à cet égard pertinent : la connaissance (au sens ordinaire du mot) est susceptible de degrés : « il y a Bismarck pour ceux qui le connaissent ; Bismarck pour ceux qui ne le connaissent qu'à travers des données historiques ; [...] Dans cette série les objets sont de plus en plus éloignés de toute expérience de particuliers. » En effet, il en va de la connaissance (presque) comme de l'amour : on peut connaître, connaître un peu, [connaître *beaucoup ; mais connaître assez/totalement], [connaître *à la folie], connaître pas du tout. D’où ce parallèle établi par Michelet (cité par le Petit Robert, connaître) : " On s'aime à mesure qu'on se connaît mieux ». En revanche, savoir - du moins dans les constructions à la $1^{\text {re }}$ personne du présent du type je sais que - ne se prête pas, en principe ${ }^{21}$, à la graduation (cf. *je sais peu que, *je ne sais pas que). Du point de vue de la théorie de la connaissance, les rôles des deux verbes discutés ici paraissent donc très différents. Dans le cas de savoir que, « la part qui revient au sujet » (cf. ci-dessus Besnier) peut se limiter à l'acceptation de la présupposition contenue dans la complétive introduite par que, alors que connaître subordonne la constitution du savoir à l'activité du sujet de connaissance.

Dans le sillage de Russell et de quelques épistémologues modernes, nous sommes donc parvenus à une vue différenciée des connaissances selon laquelle on distingue ce que certains dénomment knowledge that et knowledge of (U. Eco, 1997 : 53). Nous nous interrogerons désormais sur l'éventuelle existence de pendants lexicaux à ces deux types de connaissances, dont l'un correspondrait aux " jugements » (savoir que, knowledge that), l'autre aux propriétés d'un objet (connaître X, knowledge of).

\section{Types de noms - types de connaissances ?}

L'étude de quelques noms nous a montré plus haut (3. - 6.) que la complexité des unités lexicales génère des connaissances pouvant contribuer à la cohérence du texte. Le même constat vaut pour une partie des unités polylexicales, p. ex. les locutions verbales idiomatiques (ci-dessus, 7.). Le paragraphe 10. tente un premier aperçu de ce qui pourrait constituer l'intersection entre observations sémantiques et réflexions présentées dans le cadre des " théories de la connaissance ». C'est sur le chemin d'une telle approche que nous allons poursuivre notre recherche, en espérant qu'une convergence des deux disciplines apporte un profit pour l'une et l'autre. Leur comparabilité tient partiellement au rôle joué par l'opposition entre le concept de proposition et celui d'objet.

${ }^{21}$ Pour une présentation plus nuancée de cette question, cf. P. Blumenthal, 1999. 


\subsection{Noms « extravertis » représentant des « tranches de vie »}

Nous avons vu ci-dessus (3.) que le nom jalousie, hautement complexe d'après sa définition, peut se concevoir comme le nœud central, élément inducteur d'une petite histoire, que l'on racontera de diverses manières, par exemple comme une succession de propositions, où les notions importantes de la définition trouveraient leurs rôles sémantiques comme « actants " ou " circonstants ». Réflexion qui nous ramène au texte classique de Tesnière (1969 : 102), qui remarque à propos du centre structural de la phrase :

Le nœud verbal [...] exprime tout un petit drame. Comme un drame en effet, il comporte obligatoirement un procès, et le plus souvent des acteurs et des circonstances.

Toutefois, nos observations sur jalousie (ce mot n'étant pas « verbal ») ne se situent pas au «plan structural » (= syntaxique) de Tesnière, mais au « plan sémantique ", qui est

le domaine propre de la pensée, abstraction faite de toute expression linguistique. Il ne relève pas de la grammaire [...], mais seulement de la psychologie et de la $\operatorname{logique}^{22}$.

(L. Tesnière, $1969:$ 40)

Dans l'esprit de Tesnière, on peut considérer jalousie comme un mot susceptible de mobiliser un ensemble de représentations qui réfèrent à un "drame ». Nous proposons d'appeler mots « extravertis » (dans un sens pour ainsi dire étymologique) les unités lexicales que leurs définitions dictionnairiques présentent comme aptes à évoquer une " tranche de vie » (ci-dessus 5.), en l'occurrence une forte interaction dans un ensemble exprimé par des propositions. Cela nous ramène aux « connaissances propositionnelles » (ci-dessus 10.)

\subsection{Noms « introvertis », définis comme ensembles de propriétés}

Les dictionnaires définissent d'autres mots non pas sous l'angle de l'implication de leurs référents dans un ensemble actionnel, mais du point de vue de leurs relations qualitatives, spatiales, méronymiques, temporelles, etc. ; dans ces cas, les définitions commencent, certes, par l'indication du genre prochain, mais insistent ensuite sur les éléments du contenu, et non pas sur l'aspect « dramatique » ou interactionnel. C'est le cas des mots suivants, définis par le Petit Robert : Russell.

${ }^{22}$ Cette dernière remarque rapproche, par ailleurs, le « plan sémantique » tesniérien du projet de 
(8) azote

Corps simple, appelé autrefois nitrogène (symb. $\mathrm{N} ; \mathrm{n}^{\circ}$ at. 7 ; m. at. 14,008), gaz incolore, inodore, chimiquement peu actif, qui entre dans la composition de l'atmosphère $(4 / 5)$ et des tissus vivants, animaux et végétaux (protéines).

Dans cette définition d'un nom d'objet, relativement complexe, prévaut grammaticalement la fonction de détermination, représentée par des adjectifs et une relative. L'aspect dynamique n'apparaît qu'en dehors de la définition proprement dite à propos des composés comme cycle de l'azote, qui désigne un procès.

La détermination, qui sert à distinguer et à caractériser (mais pas, comme la prédication, à mettre en scène un « drame »), peut se faire complexe de deux manières : soit par voie additive comme dans (8), soit par enchâssement de plusieurs niveaux de déterminations comme dans l'exemple suivant :

\section{(9) asymptote}

Une droite est asymptote à une courbe au voisinage de x0 (ou de l'infini) si l'écart entre la droite et la courbe tend vers 0 quand la variable tend vers $\mathrm{x} 0$ (ou l'infini) ${ }^{23}$.

La définition présente la première condition (si...) comme dépendante d'une seconde circonstance (quand...). La définition du Petit Robert maintient le principe de l'enchâssement, mais en modifiant les circonstants (telle que... consécutif, lorsque... temporel) :

\section{(10) asymptote}

Droite telle que la distance d'un point d'une courbe à cette droite tend vers zéro lorsque le point s'éloigne sur la courbe à l'infini.

Rien n'empêche de combiner les deux types de complexité discutés dans ce paragraphe, ce qui rend le calcul du degré de complexité d'une définition d'autant plus difficile. Dans tous les cas de figures, le référent du mot à définir se présente comme l'objet d'une connaissance progressive, conformément aux idées de Russell (ci-dessus 10.). Cela dit, une réflexion poussée sur les affinités entre types de définitions, d'une part, et domaines du vocabulaire à définir (vie affective, mathématiques, droit, etc.), d'autre part, nous paraît hautement souhaitable.

\footnotetext{
${ }^{23}$ A. Deledicq (2003). Mathématiques lycée. Turin, Éditions de la Cité.
} 


\section{Conclusion}

Le mot de la fin peut être bref. Nous avons observé deux chaînes causales déclenchées par des types de « complexité » (sémantique ou extralinguistique) :

- celle qui passe du mot sémantiquement complexe à la connexité et à la cohérence du texte ;

- celle induite par une réalité extralinguistique complexe dont l'expression est facilitée et rendue rhétoriquement efficace par divers moyens (recours aux locutions, à l'abstraction, etc.).

La notion de complexité réfère à un domaine de recherche vers lequel convergent sémantique, histoire des savoirs et théories de la connaissance.

\section{Références citées}

Besnier, J.-M. (2016). Les théories de la connaissance. Paris, Presses universitaires de France.

Blay, M. (Éd.). (2003). Grand dictionnaire de la philosophie. Paris, Larousse.

Blumenthal, P. (1999). Verbes de connaissance. Verbum, 21, 7-24.

Blumenthal, P. (2014). Caractéristiques et effets de la complexité sémantique de noms d'affect. In P. Blumenthal, I. Novakova \& D. Siepmann (Éds), Les émotions dans le discours (p. 175-186). Frankfurt, Peter Lang.

Blumenthal, P. (2018). Locutions prépositionnelles «phénoménologiques ». In C. VaguerFévette (Éd.), Quand les formes prennent sens : grammaire, prépositions, constructions, système (p. 195-204). Limoges, Lambert-Lucas.

Blumenthal, P., \& Mejri, S. (2019). Redewendungen in der deutschen und französischen Pressesprache: Entwicklungen, Leistungen, Unterschiede. In M. Kauffer \& Y. Keromnes (Eds.), Theorie und Empirie in der Phraseologie (p. 295-313). Tübingen, Stauffenburg.

Cassin, B. (Éd.). (2004). Vocabulaire européen des philosophies. Paris, Seuil/Le Robert. Eco, U. (1997). Kant e l'ornitorinco. Milano, Bompiani.

Eichinger, L. (2000). Deutsche Wortbildung. Eine Einführung. Tübingen, Gunter Narr.

Gallèpe, T. (Éd.). (2016). Discours, texte et langue - La fabrique des formes du sens. Bern [e.a.], Peter Lang.

Greimas, A., \& Courtés, J. (1979). Sémiotique. Dictionnaire raisonné de la théorie du langage. Paris, Hachette.

Gross, G. (2012). Manuel d'analyse linguistique. Approche sémantico-syntaxique du lexique. Villeneuve-d'Ascq, Presses universitaires du Septentrion.

Kauffer, M. (2016). Les mots composés allemands à l'interface du lexique et du texte. In A. Lalande (1972), Vocabulaire technique et critique de la philosophie (p. 103-114). Paris, Presses universitaires de France. 
Lalande, A. (1972). Vocabulaire technique et critique de la philosophie. Paris, Presses universitaires de France. Paris, Flammarion.

Morin, E. (2005). Introduction à la pensée complexe. Paris, Seuil.

Neveu, F. (2011). Dictionnaire des sciences du langage. Paris, Armand Colin.

Predazzi, F., \& Vannuccini, V. (2007). Petit voyage dans l'âme allemande. Paris, Grasset.

Rey-Debove, J. (1971). Étude linguistique et sémiotique des dictionnaires français contemporains. The Hague, Mouton.

Robert, P., Rey-Debove, J., \& Rey, A. (Éds). (1993). Le Nouveau Petit Robert. Paris, Le Robert. (PR)

Russell, B. (1989). Problèmes de philosophie. Paris, Payot.

Soler, L. (2019). Introduction à l'épistémologie. Paris, Ellipses.

Tesnière, L. (1969). Éléments de syntaxe structurale. Paris, Klincksieck.

Trésor de la langue française informatisé. Édition électronique. ATILF - CNRS. https:// www.atilf.fr (TLFi)

Vigliocco, G., \& Vinson, D. P. (2007). Semantic representation. In M. G. Gaskell (Ed.), The Oxford Handbook of Psycholinguistics (p. 195-215). Oxford, Oxford University Press. 